\title{
Precise Asymptotics in the Law of the Iterated Logarithm under Sublinear Expectations
}

\author{
Mingzhou Xu $1 D$ and Kun Cheng \\ School of Information Engineering, Jingdezhen Ceramic Institute, Jingdezhen 333403, China \\ Correspondence should be addressed to Mingzhou Xu; mingzhouxu@whu.edu.cn
}

Received 16 December 2020; Revised 17 January 2021; Accepted 22 January 2021; Published 12 February 2021

Academic Editor: Gen Q. Xu

Copyright (C) 2021 Mingzhou Xu and Kun Cheng. This is an open access article distributed under the Creative Commons Attribution License, which permits unrestricted use, distribution, and reproduction in any medium, provided the original work is properly cited.

By an inequality of partial sum and uniform convergence of the central limit theorem under sublinear expectations, we establish precise asymptotics in the law of the iterated logarithm for independent and identically distributed random variables under sublinear expectations.

\section{Introduction}

Motivated by the work of g-expectation of Peng [1], Peng $[2,3]$ initiated the concept of the sublinear expectation space, which is a powerful tool to model the uncertainty of probability and distribution. We could consider sublinear expectation as an extension of the classical linear expectation. Peng [2, 3] constructed the basic framework, investigated basic properties, and proved the law of large number and central limit theorem under sublinear expectations. Motivated by the seminal work of Peng [2, 3], more and more limit theorems under sublinear expectation space have been established, which generalize the corresponding fundamental, important limit theorems in probability and statistics. Zhang [4-6] proved the exponential inequalities and Rosenthal's inequalities and obtained an extension of the central limit theorem and Donsker's invariance principle under sublinear expectations. Wu [7] established precise asymptotics for complete integral convergence under sublinear expectations. $\mathrm{Yu}$ and $\mathrm{Wu}$ [8] studied Marcinkiewicz-type complete convergence for weighted sums under sublinear expectations. Wu and Jiang [9] obtained a strong law of large numbers and Chover's law of the iterated logarithm under sublinear expectations. Ma and $\mathrm{Wu}$ [10] studied the limiting behavior of weighted sums of extended negatively dependent random variables under sublinear expectations. Xu and Zhang $[11,12]$ studied three series theorem for independent random variables and the law of logarithm for arrays of random variables under sublinear expectations. Chen [13] proved strong laws of large numbers for sublinear expectations. For more results about limit theorems under sublinear expectations, the interested reader could refer to the studies of $\mathrm{Hu}$ et al. [14], Fang et al. [15], Kuczmaszewska [16], Wang and Wu [17], $\mathrm{Hu}$ and Yang [18], Zhang [19], and references therein.

Precise asymptotics in the law of the iterated logarithm is one of the fundamental problems in probability theory. Many related results have been derived in the probabilistic space. Their results can be found in the work of Gut and Spătaru [20]; Zhang [21]; Xiao et al. [22]; Huang et al. [23]; Jiang and Yang [24]; Wu and Wen [25]; Xu et al. [26]; Xu [27, 28]; and Xu [29]. However, in sublinear expectations, due to the uncertainty of sublinear expectation and related capacity, the precise asymptotics in the law of the iterated logarithm under sublinear expectations have not been reported. Motivated by the work of $\mathrm{Wu}$ [7], Xiao et al. [22], Xu et al. [26], and $\mathrm{Xu}$ [29], we try to investigate precise asymptotics in the law of the iterated logarithm under sublinear expectations. The aim of this paper is to prove the precise asymptotics in the law of the iterated logarithm for independent, identically distributed random variables under sublinear expectations. The main contribution of this paper 
is that we prove an useful inequality under sublinear expectations in Lemma 1, and we extend the results of Xiao et al. [22], Xu et al. [26], and Xu [29] to those of the sublinear expectation spaces. Our results may have the potential applications in finance or engineering fields (cf. Wu [7], Peng [3], Zhang [19], and references therein). Our basic idea in this paper comes from that of $\mathrm{Wu}$ [7], Xiao et al. [22], Xu et al. [26], Xu [29], Spătaru [30], and Fuk and Nagaev [31]. In conclusion, our results combined with the work of $\mathrm{Wu}$ [7] imply heuristically that many results about precise asymptotics in the law of the iterated logarithm in probability spaces may still hold under sublinear expectations.

The rest of this paper is organized as follows: in Section 2, we summarize necessary basic notions, concepts, and relevant properties and give necessary lemmas under sublinear expectations. In Section 3, we give our main results, Theorems 1 and 2, whose proofs are presented in Sections 4 and 5 , respectively.

\section{Preliminaries}

We use notations similar to those of Peng [3]. Let $(\Omega, \mathscr{F})$ be a given measurable space. Let $\mathscr{H}$ be a subset of all random variables on $(\Omega, \mathscr{F})$ such that $I_{A} \in \mathscr{H}$, where $A \in \mathscr{F}$, and if $X_{1}, \ldots, X_{n} \in \mathscr{H}$, then $\varphi\left(X_{1}, \ldots, X_{n}\right) \in \mathscr{H}$ for each $\varphi \in C_{l, \text { Lip }}\left(\mathbb{R}^{n}\right)$, where $C_{l, \text { Lip }}\left(\mathbb{R}^{n}\right)$ denotes the linear space of (local Lipschitz) function $\varphi$ satisfying

$$
|\varphi(\mathbf{x})-\varphi(\mathbf{y})| \leq C\left(1+|\mathbf{x}|^{m}+|\mathbf{y}|^{m}\right)(|\mathbf{x}-\mathbf{y}|), \quad \forall \mathbf{x}, \mathbf{y} \in \mathbb{R}^{n},
$$

for some $C>0, m \in \mathbb{N}$, depending on $\varphi$. We regard $\mathscr{H}$ as the space of random variables.

Definition 1. A sublinear expectation $\mathbb{E}$ on $\mathscr{H}$ is a functional $\mathbb{E}: \mathbb{H} \mapsto \overline{\mathbb{R}}:=[-\infty, \infty]$ satisfying the following properties: for all $X, Y \in \mathscr{H}$, we have the following:

(a) Monotonicity: if $X \geq Y$, then $\mathbb{E}[X] \geq \mathbb{E}[Y]$

(b) Constant preserving: $\mathbb{E}[c]=c, \forall c \in \mathbb{R}$

(c) Positive homogeneity: $\mathbb{E}[\lambda X]=\lambda \mathbb{E}[X], \forall \lambda \geq 0$

(d) Subadditivity: $\mathbb{E}[X+Y] \geq \mathbb{E}[X]+\mathbb{E}[Y]$ whenever $\mathbb{E}[X]+\mathbb{E}[Y]$ is not of the form $\infty-\infty$ or $-\infty+\infty$

A set function $V: \mathscr{F} \mapsto[0,1]$ is called a capacity if it satisfies the following:

(a) $V(\varnothing)=0, V\left(\Omega_{1}\right)=1$

(b) $V(A) \leq V(B), A \subset B, A, B \in \mathscr{F}$

A capacity $V$ is said to be subadditive if it satisfies $V(A+B) \leq V(A)+V(B), A, B \in \mathscr{F}$.

In this paper, given a sublinear expectation space $(\Omega, \mathscr{H}, \mathbb{E})$, we define a capacity: $\mathbb{V}(A):=\inf \{\mathbb{E}[\xi]$ : $\left.I_{A} \leq \xi, \quad \xi \in \mathscr{H}\right\}, \forall A \in \mathscr{F}$ (see Zhang [4]). Clearly, $\mathbb{V}$ is a subadditive capacity. We also define the Choquet expectations $C_{\mathbb{V}}$ by

$$
C_{\mathbb{V}}(X):=\int_{0}^{\infty} \mathbb{V}(X>x) \mathrm{d} x+\int_{-\infty}^{0}(\mathbb{V}(X>x)-1) \mathrm{d} x .
$$

A sublinear expectation $\mathbb{E}: \mathscr{H} \mapsto \mathbb{R}$ is said to be continuous if it satisfies the following:

(a) Lower continuity: $\mathbb{E}\left[X_{n}\right] \uparrow \mathbb{E}[X]$, if $0 \leq X_{n} \uparrow X$, where $X_{n}, \quad X \in \mathscr{H}$

(b) Upper continuity: $\mathbb{E}\left[X_{n}\right] \downarrow \mathbb{E}[X]$, if $0 \leq X_{n} \downarrow X$, where $X_{n}, \quad X \in \mathscr{H}$

A capacity $V: \mathscr{F} \mapsto[0,1]$ is said to be continuous capacity if it satisfies the following:

(1) Lower continuity: $V\left(A_{n}\right) \uparrow V(A)$, if $A_{n} \uparrow A$, where $A_{n}, A \in \mathscr{F}$

(2) Upper continuity: $V\left(A_{n}\right) \downarrow V(A)$, if $A_{n} \downarrow A$, where $A_{n}, A \in \mathscr{F}$

Assume that $\mathbf{X}=\left(X_{1}, \ldots, X_{m}\right), \quad X_{i} \in \mathscr{H}, \quad$ and $\mathbf{Y}=\left(Y_{1}, \ldots, Y_{n}\right), Y_{i} \in \mathscr{H}$, are two random variables on $\left(\Omega_{1}, \mathscr{H}, \mathbb{E}\right) . \mathbf{Y}$ is said to be independent of $\mathbf{X}$ if for each $\varphi \in C_{l, \text { Lip }}\left(\mathbb{R}^{m} \times \mathbb{R}^{n}\right)$, we have $\mathbb{E}[\varphi(\mathbf{X}, \mathbf{Y})]=\mathbb{E}\left[\left.\varphi(\mathbf{x}, \mathbf{Y})\right|_{\mathbf{x}=\mathbf{X}}\right]$ whenever $\bar{\varphi}(\mathbf{x}):=\mathbb{E}[|\varphi(\mathbf{x}, \mathbf{Y})|]<\infty$ for each $\mathbf{x}$ and $\mathbb{E}[|\bar{\varphi}(\mathbf{X})|]<\infty .\left\{X_{n}\right\}_{n=1}^{\infty}$ is said to be a sequence of independent random variables, if $X_{n+1}$ is independent of $\left(X_{1}, \ldots, X_{n}\right)$ for each $n \geq 1$.

Suppose that $\mathbf{X}_{1}$ and $\mathbf{X}_{2}$ are two $n$-dimensional random vectors defined, respectively, in sublinear expectation spaces $\left(\Omega_{1}, \mathscr{H}_{1}, \mathbb{E}_{1}\right)$ and $\left(\Omega_{2}, \mathscr{H}_{2}, \mathbb{E}_{2}\right)$. They are said to be identically distributed if

$$
\mathbb{E}_{1}\left[\varphi\left(\mathbf{X}_{1}\right)\right]=\mathbb{E}_{2}\left[\varphi\left(\mathbf{X}_{2}\right)\right], \quad \forall \varphi \in C_{l, \text { Lip }}\left(\mathbb{R}^{n}\right),
$$

whenever the sublinear expectations are finite. $\left\{X_{n}\right\}_{n=1}^{\infty}$ is said to be identically distributed if for each $i \geq 1, X_{i}$ and $X_{1}$ are identically distributed.

For $0 \leq \underline{\sigma}^{2} \leq \bar{\sigma}^{2}<\infty$, a random variable $\xi$ under a sublinear expectation space $(\Omega, \mathscr{H}, \mathbb{E})$ is called a G-normal $\mathcal{N}\left(0,\left[\underline{\sigma}^{2}, \bar{\sigma}^{2}\right]\right)$ distributed random variable, if for any $\varphi \in C_{l, \text { Lip }}\left(\mathbb{R}^{n}\right), \quad u(x, t):=\mathbb{E}[\varphi(x+\sqrt{t} \xi)](x \in \mathbb{R}, t \geq 0)$ is the unique viscosity solution of the following heat equation:

$$
\begin{aligned}
\partial_{t} u-G\left(\partial_{x x}^{2} u\right) & =0, \\
u(0, x) & =\varphi(x),
\end{aligned}
$$

where $G(\alpha)=\left(\bar{\sigma}^{2} \alpha^{+}-\sigma^{2} \alpha^{-}\right) / 2$.

In the rest of this paper, let $\left\{X, X_{n}, n \geq 1\right\}$ be a sequence of i.i.d. random variables under sublinear expectation space $(\Omega, \mathscr{H}, \mathbb{E})$ with $\mathbb{E}(X)=\mathbb{E}(-X)=0, \mathbb{E}\left(X^{2}\right)=\bar{\sigma}^{2}<\infty$, and $-\mathbb{E}\left(-X^{2}\right)=\underline{\sigma}^{2}, \lim _{c \longrightarrow \infty} \mathbb{E}\left(X^{2}-c\right)^{+}=0, C_{\mathbb{V}}\left(X^{2}\right)<\infty$. Set $S_{n}=\sum_{i=1}^{n} X_{i}$. Assume that $\mathbb{E}$ is continuous. Let $\xi$ be a $G$-normal-distributed random variable with $\mathbb{E}(\xi)=\mathbb{E}(-\xi)=0, \mathbb{E}\left(\xi^{2}\right)=\bar{\sigma}^{2}$, and $-\mathbb{E}\left(-\xi^{2}\right)=\sigma^{2}$. We denote by $C$ a positive constant which may vary from line to line.

To prove our results, we need the following lemmas.

Lemma 1. Suppose $\mathbb{E}|X|^{\alpha}<\infty, 1<\alpha \leq 2$. Then, for $x, y>0$,

$$
\mathbb{V}\left\{\left|S_{n}\right| \geq x\right\} \leq 2 n \mathbb{V}\{|X|>y\}+2 n^{x / y}\left(\frac{e \mathbb{E}|X|^{\alpha}}{n \mathbb{E}|X|^{\alpha}+x y^{\alpha-1}}\right)^{x / y} \text {. }
$$


Proof. We borrow the proofs from those of Theorem 2 by Fuk and Nagaev [31], and Lemma 2 by Spătaru [30]. Let

$$
\begin{aligned}
& \widetilde{X}_{i}=\left\{\begin{array}{ccc}
X_{i} & \text { for } & \left|X_{i}\right| \leq y, \\
0 & \text { for } & \left|X_{i}\right|>y,
\end{array} \quad i=1, \ldots, n,\right. \\
& \widetilde{S}_{n}=\sum_{i=1}^{n} \widetilde{X}_{i} .
\end{aligned}
$$

Therefore, by the subadditivity property of $\mathbb{V}(\cdot)$,

$$
\mathbb{V}\left\{S_{n} \geq x\right\} \leq \mathbb{V}\left\{\widetilde{S}_{n} \neq S_{n}\right\}+\mathbb{V}\left\{\widetilde{S}_{n} \geq x\right\} .
$$

By Markov's inequality under sublinear expectations, for any positive $h$,

$$
\mathbb{V}\left\{\widetilde{S}_{n} \geq x\right\} \leq \mathrm{e}^{-h x} \mathbb{E}\left(\mathrm{e}^{h \widetilde{S}_{n}}\right) .
$$

From this and (7), it follows that

$$
\begin{aligned}
\mathbb{V}\left\{S_{n}\right. & \geq x\} \leq \sum_{i=1}^{n} \mathbb{V}\left\{\left|X_{i}\right| \geq y\right\}+\mathrm{e}^{-h x} \mathbb{E}\left(\mathrm{e}^{\hat{h S_{n}}}\right) \\
& =n \mathbb{V}\{X \geq y\}+\mathrm{e}^{-h x} \mathbb{E}\left(\mathrm{e}^{h \widetilde{S}_{n}}\right) .
\end{aligned}
$$

Application of the monotonicity of $u^{-2}\left(\mathrm{e}^{h u}-1-h u\right)$ for $u \leq y$ and $u^{-\alpha}\left(\mathrm{e}^{h u}-1-h u\right)$ for $u>0$ and the subadditivity property of sublinear expectations yields

$$
\begin{aligned}
\mathbb{E} \mathrm{e}^{h \tilde{X}_{i}} & \leq 1+\mathbb{E}\left(h X_{i} I_{\left|X_{i}\right| \leq y}\right)+\mathbb{E}\left(\frac{\mathrm{e}^{h X_{i}}-1-h X_{i}}{X_{i}^{2}} X_{i}^{2} I_{\left|X_{i}\right| \leq y}\right) \\
& \leq 1+h \mathbb{E}\left(X_{i} I_{\left|X_{i}\right| \leq y}\right)+\frac{\mathrm{e}^{h y}-1-h y}{y^{2}} \mathbb{E}\left(X_{i}^{2} I_{\left|X_{i}\right| \leq y}\right) \\
& \leq 1+h \mathbb{E}\left(X_{i} I_{\left|X_{i}\right| \leq y}\right)+\frac{\mathrm{e}^{h y}-1-h y}{y^{\alpha}} \mathbb{E}\left(\left|X_{i}\right|^{\alpha} I_{\left|X_{i}\right| \leq y}\right) \\
& \leq 1+h \mathbb{E}\left(X_{i} I_{\left|X_{i}\right| \leq y}\right)+\frac{\mathrm{e}^{h y}-1-h y}{y^{\alpha}} \mathbb{E}\left(\left|X_{i}\right|^{\alpha}\right) .
\end{aligned}
$$

Hence, by Lemma 1.1 in the study of Gao and $\mathrm{Xu}$ [32],

$$
\begin{aligned}
\mathrm{e}^{-h x} \mathbb{E}\left(\mathrm{e}^{h \tilde{S}_{n}}\right) \leq & \exp \left\{\left(\mathrm{e}^{h y}-1-h y\right) y^{-\alpha} n \mathbb{E}\left(|X|^{\alpha}\right)\right. \\
& \left.-h x+h n \mathbb{E}\left(X I_{|X| \leq y}\right)\right\} .
\end{aligned}
$$

Setting

$$
h=\frac{1}{y} \log \left(\frac{x y^{\alpha-1}}{n \mathbb{E}\left(|X|^{\alpha}\right)}+1\right),
$$

in the right-hand side of (11), we see that

$$
\mathrm{e}^{-h x} \mathbb{E}\left(\mathrm{e}^{\tilde{h S}_{n}}\right) \leq \exp \left\{\frac{x}{y}-\left(\frac{x-n \mathbb{E}\left(X I_{|X| \leq y}\right)}{y}+\frac{n \mathbb{E}\left(|X|^{\alpha}\right)}{y^{\alpha}}\right) \log \left(\frac{x y^{\alpha-1}}{n \mathbb{E}\left(|X|^{\alpha}\right)}+1\right)\right\}
$$

Since $\mathbb{E}(X)=\mathbb{E}(-X)=0$, by Proposition 3.6 in the study of Peng [3] and Definition 1, we see that

$$
\begin{aligned}
\mathbb{E}\left(X I_{|X| \leq y}\right) & =\left|\mathbb{E}\left(-X I_{|X| \geq y}\right)\right| \leq \mathbb{E}\left(|X| I_{|X| \geq y}\right) \\
& \leq \frac{1}{y^{\alpha-1}} \mathbb{E}\left(|X|^{\alpha} I_{|X| \geq y}\right) \leq \frac{1}{y^{\alpha-1}} \mathbb{E}\left[|X|^{\alpha}\right] .
\end{aligned}
$$

Therefore,

$$
\frac{1}{y}\left(x-n \mathbb{E}\left(X I_{|X| \leq y}\right)\right)+\frac{n \mathbb{E}\left(|X|^{\alpha}\right)}{y^{\alpha}} \geq \frac{x}{y} .
$$

Combining this with (13) and (9), we conclude that

$$
\mathbb{V}\left\{S_{n} \geq x\right\} \leq n \mathbb{V}\{|X|>y\}+n^{x / y}\left(\frac{\mathrm{e} \mathbb{E}|X|^{\alpha}}{n \mathbb{E}|X|^{\alpha}+x y^{\alpha-1}}\right)^{x / y} .
$$

Combining (16) with the inequality derived from it with $-X$ and $-X_{k}$ in place of $X$ and $X_{k}$, respectively, leads to (5).
Remark 1. (see Lemma 2 in [7]). For any $X \in \mathscr{H}$, we have

$$
C_{\mathbb{V}}\left(X^{2}\right)<\infty \Leftrightarrow \int_{1}^{\infty} x \mathbb{V}(|X|>x) \mathrm{d} x<\infty .
$$

Lemma 2 (see Lemma 5 in [7]). Assume that $\left\{X_{n} ; n \geq 1\right\}$ is a sequence of independent and identically distributed random variables with $\mathbb{E}\left[X_{1}\right]=\mathbb{E}\left[-X_{1}\right]=0$ and $\lim _{c \rightarrow \infty}$ $\mathbb{E}\left(X^{2}-c\right)^{+}=0$. Write $\bar{\sigma}^{2}=\mathbb{E}\left[X_{1}^{2}\right]$ and $\underline{\sigma}^{2}=-\mathbb{E}\left[-X_{1}^{2}\right]$. Suppose that $\mathbb{E}$ is continuous and set $\Delta_{n}(x)=\mathbb{V}\left(\left|S_{n}\right| /\right.$ $\sqrt{n} \geq x)-\mathbb{V}(|\xi| \geq x), \xi \sim \mathcal{N}\left(0,\left[\underline{\sigma}^{2}, \bar{\sigma}^{2}\right]\right)$ under $\mathbb{E}$. Then,

$$
\Delta_{n}:=\sup _{x \geq 0}\left|\Delta_{n}(x)\right| \longrightarrow 0, \text { as } n \longrightarrow \infty .
$$

\section{Main Results}

The following are our main results.

Theorem 1. For $b, d>0$, we have

$$
\lim _{\varepsilon \searrow 0} \varepsilon^{b / d} \sum_{n=3}^{\infty} \frac{(\log \log n)^{b-1}}{n \log n} \mathbb{V}\left\{\left|S_{n}\right| \geq \varepsilon \sqrt{n}(\log \log n)^{d}\right\}=\frac{C_{\mathbb{V}}\left(|\xi|^{b / d}\right)}{b} .
$$


Theorem 2. For $d>0$, we have

$$
\lim _{\varepsilon \searrow 0} \frac{1}{-\log \varepsilon} \sum_{n=3}^{\infty} \frac{1}{n \log n \log \log n} \mathbb{V}\left\{\left|S_{n}\right| \geq \varepsilon \sigma \sqrt{n}(\log \log n)^{d}\right\}=\frac{1}{d}
$$

In the following two sections, for $M \geq 3$ and $0<\varepsilon<1$, set $b(\varepsilon)=\left\lfloor\exp \left\{\exp \left\{M \varepsilon^{-1 / d}\right\}\right\}\right\rfloor$.

\section{Proof of Theorem 1}

Proposition 1. For $b, d>0$, we have

$$
\lim _{\varepsilon \searrow 0} \varepsilon^{b / d} \sum_{n=3}^{\infty} \frac{(\log \log n)^{b-1}}{n \log n} \mathbb{V}\left\{|\xi| \geq \varepsilon(\log \log n)^{d}\right\}=\frac{C_{\mathbb{V}}\left(|\xi|^{b / d}\right)}{b} .
$$

Proof.

$$
\begin{aligned}
& \lim _{\varepsilon \searrow 0} \varepsilon^{b / d} \sum_{n=3}^{\infty} \frac{(\log \log n)^{b-1}}{n \log n} \mathbb{V}\left\{|\xi| \geq \varepsilon(\log \log n)^{d}\right\} \\
& =\lim _{\varepsilon \searrow 0} \varepsilon^{b / d} \int_{\mathrm{e}^{\mathrm{e}}}^{\infty} \frac{(\log \log y)^{b-1}}{y \log y} \mathbb{V}\left\{|\xi| \geq \varepsilon(\log \log y)^{d}\right\} \mathrm{d} y \\
& =\lim _{\varepsilon \searrow 0} \varepsilon^{b / d} \int_{\varepsilon}^{\infty}(y / \varepsilon)^{(b-1) / d}(1 / d)(y / \varepsilon)^{1 / d-1} \frac{1}{\varepsilon} \mathbb{V}(|\xi| \geq y) \mathrm{d} y \\
& =\lim _{\varepsilon \searrow 0} \int_{\varepsilon}^{\infty}(1 / d) y^{b / d-1} \mathbb{V}(|\xi| \geq y) \mathrm{d} y \\
& =\lim _{\varepsilon \searrow 0} \int_{\varepsilon^{b / d}}^{\infty}(1 / b) \mathbb{V}\left(|\xi|^{b / d} \geq t\right) \mathrm{d} t \\
& =\int_{0}^{\infty}(1 / b) \mathbb{V}\left(|\xi|^{b / d} \geq t\right) \mathrm{d} t \\
& =\frac{C_{\mathbb{V}}\left(|\xi|^{b / d}\right)}{b} \text {. }
\end{aligned}
$$

Thus, this completes the proof of Proposition 1 .

Remark 2. By the proof of (24) and (25) in the study by Wu [7], $C_{\mathbb{V}}\left(|\xi|^{b / d}\right)$ is finite for any $b, d>0$.

Proposition 2. For $b, d>0$, we have

$$
\begin{gathered}
\lim _{\varepsilon \searrow 0} \varepsilon^{b / d} \sum_{n \leq b(\varepsilon)} \frac{(\log \log n)^{b-1}}{n \log n} \mid \mathbb{V}\left\{\left|S_{n}\right| \geq \varepsilon \sqrt{n}(\log \log n)^{d}\right\} \\
-\mathbb{V}\left\{|\xi| \geq \varepsilon(\log \log n)^{d}\right\} \mid=0 .
\end{gathered}
$$

Proof. By Lemma 2 and Toeplitz's lemma,

$$
\lim _{\varepsilon \searrow 0} \varepsilon^{b / d} \sum_{n \leq b(\varepsilon)} \frac{(\log \log n)^{b-1}}{n \log n} \mid \mathbb{V}\left\{\left|S_{n}\right| \geq \varepsilon \sqrt{n}(\log \log n)^{d}\right\}
$$

$$
\begin{gathered}
-\mathbb{V}\left\{|\xi| \geq \varepsilon(\log \log n)^{d}\right\} \mid \\
\leq \lim _{\varepsilon \searrow 0} \varepsilon^{b / d} \sum_{n \leq b(\varepsilon)} \frac{(\log \log n)^{b-1}}{n \log n} \Delta_{n}
\end{gathered}
$$

$$
=\lim _{\varepsilon \searrow 0} \frac{C M^{b}}{(\log \log (b(\varepsilon)))^{b}} \sum_{n \leq b(\varepsilon)} \frac{(\log \log n)^{b-1}}{n \log n} \Delta_{n}=0 .
$$

The proof is complete.

Proposition 3. For $b, d>0$, we have

$$
\lim _{M \longrightarrow \infty} \limsup _{\varepsilon \searrow 0} \varepsilon^{b / d} \sum_{n>b(\varepsilon)} \frac{(\log \log n)^{b-1}}{n \log n} \mathbb{V}\left\{|\xi| \geq \varepsilon(\log \log n)^{d}\right\}=0 .
$$

Proof. We could obtain that

$$
\begin{aligned}
& \underset{\varepsilon \searrow 0}{\limsup } \varepsilon^{b / d} \sum_{n>b(\varepsilon)} \frac{(\log \log n)^{b-1}}{n \log n} \mathbb{V}\left\{|\xi| \geq \varepsilon(\log \log n)^{d}\right\} \\
& \quad \leq C \underset{\varepsilon \searrow 0}{\limsup \varepsilon^{b / d}} \int_{b(\varepsilon)}^{\infty} \frac{(\log \log y)^{b-1}}{y \log y} \mathbb{V}\left\{|\xi| \geq \varepsilon(\log \log y)^{d}\right\} \mathrm{d} y \\
& \quad \leq C \int_{M^{d}}^{\infty} t^{b / d-1} \mathbb{V}\{|\xi| \geq t\} \mathrm{d} t \\
& \quad=C \int_{M^{b}}^{\infty} \mathbb{V}\left\{|\xi|^{b / d} \geq t\right\} \mathrm{d} t .
\end{aligned}
$$

Note that $\int_{M^{b}}^{\infty} \mathbb{V}\left\{|\xi|^{b / d} \geq t\right\} \mathrm{d} t$ is integrable:

$$
\int_{M^{b}}^{\infty} \mathbb{V}\left\{|\xi|^{b / d} \geq t\right\} \mathrm{d} t \longrightarrow 0
$$

as $M \longrightarrow \infty$. Proposition 3 is established. 
Proposition 4. For $b, d>0$, we have

$$
\lim _{M \longrightarrow \infty} \limsup _{\varepsilon \succ 0} \varepsilon^{b / d} \sum_{n>b(\varepsilon)} \frac{(\log \log n)^{b-1}}{n \log n} \mathbb{V}\left\{\left|S_{n}\right| \geq \varepsilon \sqrt{n}(\log \log n)^{d}\right\}=0
$$

Proof. When $0<b<2 d$, by Markov's inequality under sublinear expectations, we have

$$
\begin{aligned}
& \limsup _{\varepsilon \supset 0} \varepsilon^{b / d} \sum_{n>b(\varepsilon)} \frac{(\log \log n)^{b-1}}{n \log n} \mathbb{V}\left\{\left|S_{n}\right| \geq \varepsilon \sqrt{n}(\log \log n)^{d}\right\} \\
& \leq C \limsup _{\varepsilon \downarrow 0} \varepsilon^{b / d-2} \sum_{n>b(\varepsilon)} \frac{(\log \log n)^{b-1-2 d}}{n^{2} \log n} \mathbb{E}\left[S_{n}^{2}\right] \\
& =C \limsup _{\varepsilon \downarrow 0} \varepsilon^{b / d-2} \sum_{n>b(\varepsilon)} \frac{(\log \log n)^{b-1-2 d}}{n \log n} \\
& \leq C \limsup _{\varepsilon \downarrow 0} \varepsilon^{b / d-2}(\log \log (b(\varepsilon)))^{b-2 d} \\
& \leq C M^{b-2 d} \longrightarrow 0 \text {, as } M \longrightarrow \infty \text {. }
\end{aligned}
$$

For $b \geq 2 d$, by Lemma 1 , we see that

$$
\begin{aligned}
& \sum_{n>b(\varepsilon)} \frac{(\log \log n)^{b-1}}{n \log n} \mathbb{V}\left\{\left|S_{n}\right| \geq \varepsilon \sqrt{n}(\log \log n)^{d}\right\} \\
& \leq \sum_{n>b(\varepsilon)} \frac{(\log \log n)^{b-1}}{\log n} \mathbb{V}\left\{|X|>\varepsilon \sqrt{n}(\log \log n)^{d} / T\right\} \\
& +C \sum_{n>b(\varepsilon)} \frac{(\log \log n)^{b-1}}{n \log n} \frac{1}{(\log \log n)^{2 d T} \varepsilon^{2 T}}=: L_{1}+L_{2},
\end{aligned}
$$

where $T$ is a positive constant to be specified later. On the one hand, we obtain that

$$
\begin{aligned}
\limsup _{\varepsilon \searrow 0} \varepsilon^{b / d} L_{2} & \leq \underset{\varepsilon \searrow 0}{\limsup \varepsilon^{b / d-2 T}} \sum_{n>b(\varepsilon)} \frac{(\log \log n)^{b-1-2 d T}}{n \log n} \\
& \leq \underset{\varepsilon \searrow 0}{\limsup C \varepsilon^{b / d-2 T}}(\log \log b(\varepsilon))^{b-2 d T} \\
& \leq C M^{b-2 d T} \longrightarrow 0, \text { as } M \longrightarrow \infty
\end{aligned}
$$

for any $T>b /(2 d)$. On the other hand, for $L_{1}$, without loss of generality, set $T=1$. By the countable subadditivity property of sublinear expectations and the fact that $\left((\log \log x)^{b-1} / \log x\right) \longrightarrow 0$, as $x>b(\varepsilon) \longrightarrow \infty$, we obtain that

$$
\begin{aligned}
L_{1} & =\sum_{n>b(\varepsilon)} \frac{(\log \log n)^{b-1}}{\log n} \mathbb{V}\left\{|X|>\varepsilon \sqrt{n}(\log \log n)^{d}\right\} \\
& \leq C \int_{x>b(\varepsilon)} \frac{(\log \log x)^{b-1}}{\log x} \mathbb{V}\left\{|X|>\varepsilon \sqrt{x}(\log \log x)^{d}\right\} \mathrm{d} x \\
& \leq C \int_{x>b(\varepsilon)} \mathbb{V}\left\{|X|^{2}>\varepsilon^{2} x(\log \log x)^{2 d}\right\} \mathrm{d} x \\
& \leq C \varepsilon^{-2} \int_{x>b(\varepsilon) M^{2 d}} \mathbb{V}\left\{|X|^{2}>y\right\} \mathrm{d} y .
\end{aligned}
$$

Hence, for $b \geq 2 d$, we have

$$
\limsup _{\varepsilon \searrow 0} \varepsilon^{b / d} L_{1} \leq C \varepsilon^{b / d-2} \int_{x>b(\varepsilon) M^{2 d}} \mathbb{V}\left\{|X|^{2}>y\right\} \mathrm{d} y=0 .
$$

Thus, (28) holds for each $b, d>0$.

Now, by Proposition 1-4 and the triangle inequality, $\forall \beta>0, \exists M>0$, which is sufficiently large, such that 


$$
\begin{aligned}
& \lim _{\varepsilon \supset 0} \varepsilon^{b / d} \sum_{n=3}^{\infty} \frac{(\log \log n)^{b-1}}{n \log n} \mathbb{V}\left\{\left|S_{n}\right| \geq \varepsilon \sqrt{n}(\log \log n)^{d}\right\} \\
& \leq \lim _{\mathcal{E} \backslash 0} \varepsilon^{b / d} \sum_{n=3}^{\infty} \frac{(\log \log n)^{b-1}}{n \log n} \mathbb{V}\left\{|\xi| \geq \varepsilon(\log \log n)^{d}\right\} \\
& +\lim _{\varepsilon \searrow 0} \varepsilon^{b / d} \sum_{n \leq b(\varepsilon)} \frac{(\log \log n)^{b-1}}{n \log n}\left|\mathbb{V}\left\{\left|S_{n}\right| \geq \varepsilon \sqrt{n}(\log \log n)^{d}\right\}-\mathbb{V}\left\{|\xi| \geq \varepsilon(\log \log n)^{d}\right\}\right| \\
& +\limsup _{\varepsilon \searrow 0} \varepsilon^{b / d} \sum_{n>b(\varepsilon)} \frac{(\log \log n)^{b-1}}{n \log n} \mathbb{V}\left\{|\xi| \geq \varepsilon(\log \log n)^{d}\right\} \\
& +\limsup _{\varepsilon \downarrow 0} \varepsilon^{b / d} \sum_{n>b(\varepsilon)} \frac{(\log \log n)^{b-1}}{n \log n} \mathbb{V}\left\{\left|S_{n}\right| \geq \varepsilon \sqrt{n}(\log \log n)^{d}\right\} \\
& =\frac{C_{\mathbb{V}}\left(|\xi|^{b / d}\right)}{b}+\beta \\
& \lim _{\varepsilon \searrow 0} \varepsilon^{b / d} \sum_{n=3}^{\infty} \frac{(\log \log n)^{b-1}}{n \log n} \mathbb{V}\left\{\left|S_{n}\right| \geq \varepsilon \sqrt{n}(\log \log n)^{d}\right\} \\
& \geq \lim _{\varepsilon \searrow 0} \varepsilon^{b / d} \sum_{n=3}^{\infty} \frac{(\log \log n)^{b-1}}{n \log n} \mathbb{V}\left\{|\xi| \geq \varepsilon(\log \log n)^{d}\right\} \\
& -\lim _{\varepsilon \searrow 0} \varepsilon^{b / d} \sum_{n \leq b(\varepsilon)} \frac{(\log \log n)^{b-1}}{n \log n}\left|\mathbb{V}\left\{\left|S_{n}\right| \geq \varepsilon \sqrt{n}(\log \log n)^{d}\right\}-\mathbb{V}\left\{|\xi| \geq \varepsilon(\log \log n)^{d}\right\}\right| \\
& -\limsup _{\varepsilon \searrow 0} \varepsilon^{b / d} \sum_{n>b(\varepsilon)} \frac{(\log \log n)^{b-1}}{n \log n} \mathbb{V}\left\{|\xi| \geq \varepsilon(\log \log n)^{d}\right\} \\
& -\limsup _{\varepsilon \downarrow 0} \varepsilon^{b / d} \sum_{n>b(\varepsilon)} \frac{(\log \log n)^{b-1}}{n \log n} \mathbb{V}\left\{\left|S_{n}\right| \geq \varepsilon \sqrt{n}(\log \log n)^{d}\right\} \\
& \geq \frac{C_{\mathbb{V}}\left(|\xi|^{b / d}\right)}{b}-\beta
\end{aligned}
$$

We derive Theorem 1 from the arbitrariness of $\beta>0 . \quad \square \quad \lim _{\varepsilon \searrow 0} \frac{1}{-\log \varepsilon} \sum_{n=3}^{\infty} \frac{1}{n \log n \log \log n} \mathbb{V}\left\{|\xi| \geq \varepsilon(\log \log n)^{d}\right\}$

\section{Proof of Theorem 2}

Proposition 5. For $d>0$, we have

$$
\lim _{\varepsilon \downarrow 0} \frac{1}{-\log \varepsilon} \sum_{n=3}^{\infty} \frac{1}{n \log n \log \log n} \mathbb{V}\left\{|\xi| \geq \varepsilon(\log \log n)^{d}\right\}=\frac{1}{d} .
$$

$$
\begin{aligned}
& =\lim _{\varepsilon \searrow 0} \frac{1}{-\log \varepsilon} \int_{\mathrm{e}^{\mathrm{e}}}^{\infty} \frac{1}{y \log y \log \log y} \mathbb{V}\left\{|\xi| \geq \varepsilon(\log \log y)^{d}\right\} \mathrm{d} y \\
& =\frac{1}{d} \lim _{\varepsilon \searrow 0} \frac{1}{-\log \varepsilon} \int_{\varepsilon}^{\infty} \frac{1}{t} \mathbb{V}\{|\xi| \geq t\} \mathrm{d} t \\
& =\frac{1}{d} .
\end{aligned}
$$

Proof. We claim that

Indeed, by Lemma 4 in the study by $\mathrm{Wu}$ [7], $\forall \alpha>0$, $\exists \delta>0$, such that $\forall t<\delta<1, \mathbb{V}\{|\xi|>t\}>1-\alpha d$. Therefore, 


$$
\begin{aligned}
& \frac{1}{d}\left|\lim _{\varepsilon \searrow 0} \frac{1}{-\log \varepsilon} \int_{\varepsilon}^{\infty} \frac{1}{t} \mathbb{V}\{|\xi| \geq t\} \mathrm{d} t-1\right| \\
& \quad \leq \frac{1}{d}\left|\lim _{\varepsilon \searrow 0} \frac{1}{-\log \varepsilon} \int_{\varepsilon}^{\delta} \frac{1}{t} \mathbb{V}\{|\xi| \geq t\} \mathrm{d} t-1\right|+\lim _{\varepsilon \searrow 0}\left|\frac{1}{-\log \varepsilon} \int_{\delta}^{\infty} \frac{1}{t} \mathbb{V}\{|\xi| \geq t\} \mathrm{d} t\right| \\
& \quad<\frac{1}{d} \lim _{\varepsilon \searrow 0}\left|\frac{1}{-\log \varepsilon} \int_{\varepsilon}^{\delta} \frac{1}{t}(1-\alpha d) \mathrm{d} t-1\right|+\lim _{\varepsilon \searrow 0}\left|\frac{1}{-\log \varepsilon} \int_{\delta}^{\infty} \frac{1}{t^{3}} \mathbb{E}\left[|\xi|^{2}\right] \mathrm{d} t\right| \\
& \quad \leq \alpha .
\end{aligned}
$$

This establishes (35).

Proposition 6. For $d>0$, we have

$$
\lim _{\varepsilon \backslash 0} \frac{1}{-\log \varepsilon} \sum_{n \leq b(\varepsilon)} \frac{1}{n \log n \log \log n}\left|\mathbb{V}\left\{\left|S_{n}\right| \geq \varepsilon \sqrt{n}(\log \log n)^{d}\right\}-\mathbb{V}\left\{|\xi| \geq \varepsilon(\log \log n)^{d}\right\}\right|=0
$$

Proof. By Lemma 2 and Toeplitz's lemma,

$$
\begin{aligned}
& \lim _{\varepsilon \searrow 0} \frac{1}{-\log \varepsilon} \sum_{n \leq b(\varepsilon)} \frac{1}{n \log n \log \log n}\left|\mathbb{V}\left\{\left|S_{n}\right| \geq \varepsilon \sqrt{n}(\log \log n)^{d}\right\}-\mathbb{V}\left\{|\xi| \geq \varepsilon(\log \log n)^{d}\right\}\right| \\
& \quad=\lim _{\varepsilon \searrow 0} \frac{1}{-\log \varepsilon} \sum_{n \leq b(\varepsilon)} \frac{1}{n \log n \log \log n} \Delta_{n} \\
& \quad=\lim _{\varepsilon \searrow 0} \frac{\log (M)-\log (\varepsilon) / d}{-\log \varepsilon} \frac{1}{\log \log \log b(\varepsilon)} \sum_{n \leq b(\varepsilon)} \frac{1}{n \log n \log \log n} \Delta_{n}=0 .
\end{aligned}
$$

The proof is complete.

Proposition 7. For $d>0$, we have

Proof. By Markov inequality under sublinear expectations, we see that

$\lim _{\varepsilon \searrow 0} \frac{1}{-\log \varepsilon} \sum_{n>b(\varepsilon)} \frac{1}{n \log n \log \log n} \mathbb{V}\left\{|\xi| \geq \varepsilon(\log \log n)^{d}\right\}=0$.

$$
\begin{aligned}
& \lim _{\varepsilon \searrow 0} \frac{1}{-\log \varepsilon} \sum_{n>b(\varepsilon)} \frac{1}{n \log n \log \log n} \mathbb{V}\left\{|\xi| \geq \varepsilon(\log \log n)^{d}\right\} \\
& \leq C \lim _{\varepsilon \searrow 0} \frac{1}{-\log \varepsilon} \int_{b(\varepsilon)}^{\infty} \frac{1}{y \log y \log \log y} \mathbb{V}\left\{|\xi| \geq \varepsilon(\log \log y)^{d}\right\} \mathrm{d} y \\
& \leq C \lim _{\varepsilon \searrow 0} \frac{1}{-\log \varepsilon} \int_{M^{d}}^{\infty} \frac{1}{t} \mathbb{V}\{|\xi|>t\} \mathrm{d} t \\
& \leq C \lim _{\varepsilon \unlhd 0} \frac{1}{-\log \varepsilon} \int_{M^{d}}^{\infty} \frac{1}{t^{3}} \mathbb{E}\left[\xi^{2}\right] \mathrm{d} t=0 .
\end{aligned}
$$


Thus this proves Proposition 7.

Proposition 8. For $d>0$, we have

$$
\lim _{\varepsilon \searrow 0} \frac{1}{-\log \varepsilon} \sum_{n>b(\varepsilon)} \frac{1}{n \log n \log \log n} \mathbb{V}\left\{\left|S_{n}\right| \geq \varepsilon \sqrt{n}(\log \log n)^{d}\right\}=0
$$

Proof. By Markov inequality under sublinear expectations,

we deduce that

$$
\begin{aligned}
& \lim _{\varepsilon \searrow 0} \frac{1}{-\log \varepsilon} \sum_{n>b(\varepsilon)} \frac{1}{n \log n \log \log n} \mathbb{V}\left\{\left|S_{n}\right| \geq \varepsilon \sqrt{n}(\log \log n)^{d}\right\} \\
& \leq \lim _{\varepsilon \searrow 0} \frac{1}{-\varepsilon^{2} \log \varepsilon} \sum_{n>b(\varepsilon)} \frac{1}{n^{2} \log n(\log \log n)^{1+2 d}} \mathbb{E}\left[S_{n}^{2}\right] \\
& \leq C \lim _{\varepsilon \searrow 0} \frac{1}{-\varepsilon^{2} \log \varepsilon} \sum_{n>b(\varepsilon)} \frac{1}{n \log n(\log \log n)^{1+2} d} \\
& \leq C \lim _{\varepsilon \searrow 0} \frac{(\log \log (b(\varepsilon)))^{-2 d}}{-\varepsilon^{2} \log \varepsilon} \\
& \leq C \lim _{\varepsilon \searrow 0} \frac{M^{-2 d}}{-\log \varepsilon}=0 .
\end{aligned}
$$

The proof is complete.

Finally, similar to the proof of Theorem 1, by the triangle inequality and Propositions 5-8, we finish the proof of Theorem 2.

\section{Data Availability}

No data were used to support this study.

\section{Conflicts of Interest}

The authors declare that they have no conflicts of interest.

\section{Acknowledgments}

This research was supported by the Doctoral Scientific Research Starting Foundation of Jingdezhen Ceramic Institute (no. 102/01003002031), Scientific Program of Department of Education of Jiangxi Province of China (no. GJJ190732), and National Natural Science Foundation of China (no. 61662037).

\section{References}

[1] S. G. Peng, "BSDE and related g-expectation," in Backward Stochastic Differential Equations, N. El Karoui and L. Mazliak, Eds., vol. 364 Pitman Research Notes in Mathematics Series, pp. 141-159, Longman Harlow, Harlow, UK, 1997.

[2] S. Peng, "G-expectation, G-brownian motion and related stochastic calculus of itô type," Stochastic Analysis and Applications, vol. 2, no. 4, pp. 541-567, 2007.
[3] S. G. Peng, "Nonlinear expectations and stochastic calculus under uncertainty," 2010, https://arxiv.org/abs/1002.4546.

[4] L. Zhang, "Exponential inequalities under the sub-linear expectations with applications to laws of the iterated logarithm," Science China Mathematics, vol. 59, no. 12, pp. 2503-2526, 2016.

[5] L. X. Zhang, "Rosenthal's inequalities for independent and negatively dependent random variables under sub-linear expectations with applications," Science China Mathematics, vol. 59, no. 4, pp. 759-768, 2016.

[6] L.-X. Zhang, "Donsker's invariance principle under the sublinear expectation with an application to Chung's law of the iterated logarithm," Communications in Mathematics and Statistics, vol. 3, no. 2, pp. 187-214, 2015.

[7] Q. Wu, "Precise asymptotics for complete integral convergence under sublinear expectations," Mathematical Problems in Engineering, vol. 2020, Article ID 3145935, 13 pages, 2020.

[8] D. L. Yu and Q. Y. Wu, "Marcinkiewicz type complete convergence for weighted sums under sub-linear expectations," Journal of University of Science and Technology of China, vol. 48, no. 2, pp. 89-96, 2018.

[9] Q. Wu and Y. Jiang, "Strong law of large numbers and Chover's law of the iterated logarithm under sub-linear expectations," Journal of Mathematical Analysis and Applications, vol. 460, no. 1, pp. 252-270, 2018.

[10] X. C. Ma and Q. Y. Wu, "Limiting behavior of weighted sums of extended negatively dependent random variables under sublinear expectations," Advance in Mathematics (China), vol. 48, no. 2, pp. 89-96, 2018.

[11] J. P. Xu and L. X. Zhang, "Three series theorem for independent random variables under sub-linear expectations with 
applications," Acta Mathematica Sinica, English Series, vol. 35, no. 2, pp. 172-184, 2019.

[12] J.-p. Xu and L.-x. Zhang, "The law of logarithm for arrays of random variables under sub-linear expectations," Acta Mathematicae Applicatae Sinica, English Series, vol. 36, no. 3, pp. 670-688, 2020.

[13] Z. Chen, "Strong laws of large numbers for sub-linear expectations," Science China Mathematics, vol. 59, no. 5, pp. 945-954, 2016.

[14] F. Hu, Z. J. Chen, and D. F. Zhang, "How big are the increments of-Brownian motion," Science China Mathematics, vol. 57, no. 8, pp. 1686-1700, 2014.

[15] X. Fang, S. G. Peng, Q. M. Shao, and Y. S. Song, "Limit theorems with rate of convergence under sublinear expectations," 2018, https://arxiv.org/abs/1711.10649v2.

[16] A. Kuczmaszewska, "Complete convergence for widely acceptable random variables under the sublinear expectations," Journal of Mathematical Analysis and Applications, vol. 484, no. 1, Article ID 123662, 2020.

[17] W. J. Wang and Q. Y. Wu, "Almost sure convergence of weighted sums for extended negatively dependent random variables under sub-linear expectations," Mathematica Applicata, vol. 32, no. 2, pp. 382-391, 2019.

[18] Z.-C. Hu and Y.-Z. Yang, "Some inequalities and limit theorems under sublinear expectations," Acta Mathematicae Applicatae Sinica, English Series, vol. 33, no. 2, pp. 451-462, 2017.

[19] L. X. Zhang, "Strong limit theorems for extended independent and extended negatively random variables under non-linear expectations," 2016, https://arxiv.org/abs/1608.0071v1.

[20] A. Gut and A. Spătaru, "Precise asymptotics in the law of the iterated logarithm," The Annals of Probability, vol. 28, no. 4, pp. 1870-1883, 2000.

[21] L. X. Zhang, "Precise rates in the law of the iterated logarithm," 2006, https://arxiv.org/abs/0610519v1.

[22] X.-Y. Xiao, L.-X. Zhang, and H.-W. Yin, "Precise rates in the generalized law of the iterated logarithm," Statistics of Probability Letters, vol. 83, no. 2, pp. 616-623, 2013.

[23] W. Huang, L. Zhang, and Y. Jiang, "Precise rate in the law of iterated logarithm for-mixing sequence," Applied Mathematics-A Journal of Chinese Universities Series B, vol. 18, no. 4 , pp. 482-488, 2003.

[24] C. Jiang and X. Yang, "Precise asymptotics in self-normalized sums of iterated logarithm for multidimensionally indexed random variables," Applied Mathematics-A Journal of Chinese Universities, vol. 22, no. 1, pp. 87-94, 2007.

[25] $\mathrm{H}$. Wu and J. Wen, "Precise rates in the law of the iterated logarithm for R/S statistics," Applied Mathematics-A Journal of Chinese Universities, vol. 21, no. 4, pp. 461-466, 2006.

[26] M. Xu, Y. Ding, and Y. Zhou, "Precise rates in the generalized law of the iterated logarithm in," Journal of Mathematical Research with Applications, vol. 38, no. 1, pp. 103-110, 2018.

[27] $\mathrm{M} . \mathrm{Xu}$, "Precise rates in the generalized law of the iterated logarithm in the Hilbert space," preprint, 2020.

[28] M. Xu, "Precise asymptotics for partial sums of-mixing sequence," preprint, 2020.

[29] M. Xu, "Precise rates in the generalized law of the iterated logarithm in the multidimensional space," Accepted by Chinese Journal of Applied Probability and Statistics, 2020.

[30] A. Spătaru, "Precise asymptotics in Spitzer'law of large number," Theory of Probability \& Its Applications, vol. 12, no. 3, pp. 811-819, 1999.
[31] D. K. Fuk and S. V. Nagaev, "Probability inequalities for sums of independent random variables," Theory of Probability \& Its Applications, vol. 16, no. 4, pp. 643-660, 1971.

[32] F. Q. Gao and M. Z. Xu, "Large deviations and moderate deviations for independent random variables under sublinear expectations," Science China Mathematics, vol. 41, no. 4, pp. 337-352, 2011, in Chinese. 